\title{
MUJERES Y EDUCACIÓN UNIVERSITARIA: \\ LAS AUSENTES
}

Adelina Rodríguez Pacios

Universidad de León

\section{Las mujeres y el sistema educativo}

Es innegable que en los últimos años se ha producido un aumento del nivel formativo de las mujeres. Las familias españolas reconocen la formación como un factor de movilidad social y de promoción personal. Ya no está tan extendida la práctica de proporcionar estudios a los hijos en detrimento de la formación de las hijas.

La escolarización de la mujer es mayor que la del varón en todas las edades entre los 16 y los 29 años. Incluso en el nivel universitario el número de mujeres supera al de varones. Según el último informe de la OCDE sobre educación en el mundo desarrollado, titulado PISA 2003, las mujeres están por delante de los hombres en todos los niveles del sistema educativo. En las etapas obligatorias hay más mujeres por razones demográficas. Pero los datos de la OCDE reflejan también que las mujeres tienen más confianza en su futuro. De hecho, según datos del CIS del año 2000, un 43.5\% de las mujeres de 15 años desean llegar a terminar unos estudios universitarios, ya sean medios o superiores. En el año 2001 había 781.236 mujeres matriculadas en las universidades españolas, casi 110.000 más que hombres. (Mari-Klose, Nos Colom, 1999: 12-14; EL PAIS, 5-X-03).

Pero el sistema educativo y el familiar siguen fomentando las desigualdades. Lidia Falcón, en su libro Violencia contra la mujer, en el capítulo IX, hace referencia a un informe elaborado por el ministerio de Educación y 
Ciencia en el año 1989, con el título Guía didáctica para una educación no sexista, en el que se pone en evidencia este hecho: "La discriminación sexual comienza en la escuela y en la familia, y en ambas instituciones los adultos que las dirigen, profesores y padres (en especial el padre), son los que imponen las normas y rigen la educación de niños y niñas" (Falcón, 1991: 213).

La razón de prolongar los estudios está en la esperanza de encontrar un buen empleo. Según datos de la Encuesta de Población Activa (INE) referidos al segundo trimestre de 2003, se percibe cómo a medida que aumenta el nivel educativo, se incrementa el número de ocupadas. Así, por ejemplo, entre las mujeres que tienen estudios primarios, están ocupadas un $15.6 \%$ frente a un $42 \%$ de varones. Entre aquellos que tienen una "educación secundaria generalista", el $41 \%$ son mujeres frente al $69.9 \%$ de varones. Si consideramos la categoría "enseñanzas profesionales", vemos que un $62.2 \%$ de las mujeres aquí situadas están ocupadas frente a un $82.2 \%$ de varones. Entre aquellas que tienen titulación universitaria, el 71.6\% tienen un trabajo remunerado frente al 76.9\% de sus compañeros. Podemos apreciar también cómo las diferencias ocupacionales entre hombres y mujeres se van reduciendo a medida que aumenta la cualificación (CES, 3/2003: 81).

Son las familias de clase media y clase alta las que más animan a sus hijas a prolongar su formación. Las hijas tienden a reproducir los estudios de las madres. Hay mayor proporción de hijas con estudios universitarios que, a su vez, las madres tienen estudios universitarios (Marí-Klose, Nos Colom, 1999: 23).

Existe cada vez mayor participación de las mujeres en las distintas organizaciones, pero ésta se reduce a los niveles inferiores e intermedios. Son muy pocas las que alcanzan los niveles superiores, los niveles en los que se toman decisiones que más tarde afectan a las propias mujeres. Es lo que se ha llamado el "techo de cristal". Es decir, el crecimiento educativo femenino no ha ido acompañado de un incremento paralelo en el acceso a los puestos 
mejor pagados y más prestigiosos (Ministerio de Cultura. Instituto de la Mujer, 1985). En la Encuesta de Población Activa (INE), referida al segundo trimestre del año 2000, podemos comprobar cómo un 1.9\% de mujeres ocupan un puesto de dirección de empresas y de la Administración Pública. Sin embargo, se encuentran en la misma situación un $2.6 \%$ de hombres. Si este hecho lo analizamos en función del nivel educativo, observamos que de las mujeres que tienen estudios universitarios, son directivas un $2.1 \%$, mientras que entre los varones universitarios este número se duplica (CES, 3/2003: 90). Asimismo, esta situación la podemos encontrar en el ámbito universitario. En unas declaraciones a un periódico de tirada nacional, la profesora de la UNED Marisa García de Cortázar señalaba que el éxito académico femenino no se hace notar entre el profesorado universitario. "Cuanto más subes en el escalafón, menos hay. Entre los alumnos hay mayoría de mujeres. Luego, sólo son un $40 \%$ de los profesores asociados y un 33\% de los titulares. Por último, sólo hay un $12 \%$ de catedráticas". Algo que debemos resaltar es que de 50 universidades públicas y privadas que hay en España, sólo hay cuatro mujeres rectoras (EL PAÍS, 5-X-03).

La educación es percibida por las mujeres como un buen pasaporte para disminuir la desigualdad existente en nuestra organización social: las mayores tasas de escolarización de la población son femeninas, el rendimiento escolar femenino es notablemente superior, las mujeres activas ocupadas tienen mayor nivel de estudios que los varones, etc. Incluso se ha podido observar que a medida que se incrementa el nivel formativo de las mujeres, se incrementa su tasa de actividad. Además el comportamiento laboral de las mujeres con estudios superiores es similar al de los hombres, lo que indica que la cualificación es un instrumento de igualación en los comportamientos laborales de hombres y mujeres (Centro de Estudios del Cambio Social, 1999). Esto quiere decir que cuanto mayor es el nivel de estudios de las mujeres, menor es la diferencia en cuanto al nivel de ocupación entre hombres 
y mujeres. Así, nuevamente, si repasamos las cifras de la Encuesta de Población Activa del segundo trimestre del año 2003, comprobamos cómo la diferencia porcentual entre hombres y mujeres ocupados con una "educación secundaria generalista" es casi de 29 puntos, en la categoría de "enseñanzas profesionales" es de 20 puntos y en la de titulación universitaria es de poco más de 5 puntos (CES, 3/2003:81).

Pero seguimos encontrando diferencias significativas en el tipo de estudios que eligen las mujeres. Como se ha comentado anteriormente, este hecho pone en evidencia la influencia de las familias y de la escuela, que llevan a cabo, de manera inconsciente, un proceso de socialización que determina el futuro de los chicos y de las chicas. "La actitud discriminatoria empieza en la misma familia, donde aún se valora de forma diferente la enseñanza de los hijos y de las hijas. La de éstas se considera de menor alcance, inclusive por la propia madre, que ha sido educada en tales principios" (Sarries Sanz, 1993: $320)$.

Más de la mitad de los alumnos matriculados en enseñanzas no universitarias son mujeres. Pero la elección de estudios comienza a edades muy tempranas. De hecho, al finalizar la enseñanza obligatoria las mujeres siguen siendo mayoría, excepto en los estudios de Formación Profesional, hacia donde se siguen dirigiendo en mayor medida los hombres. Según datos del Instituto de la Mujer, para el curso 1998-99, el 52,91\% de los alumnos matriculados en el Bachillerato eran mujeres. Sin embargo, en los estudios de Formación Profesional el 52.90\% son varones. Comparando estas últimas cifras con las de cursos anteriores, se comprueba un ligero cambio en el caso de la Formación Profesional, ya que va incrementándose lentamente la presencia de las mujeres, sobre todo en aquellas ramas tipificadas como masculinizadas (Las Mujeres en cifras, 2001).

También se aprecian diferencias significativas en la elección de Bachillerato. Estas elecciones tienen una gran incidencia en el futuro académico 
y profesional de los y las jóvenes. Los estudios universitarios van a depender de esta inclinación académica. En todas las modalidades de Bachiller, menos en la modalidad de Tecnología, la presencia de las mujeres es superior a la de los hombres. Las alumnas se concentran, sobre todo, en el Bachiller de Humanidades y Ciencias Sociales (CIDE/Instituto de la Mujer, 2001: 43).

Estas decisiones van a marcar las trayectorias profesionales del alumnado. Así encontramos que las alumnas se dirigen hacia carreras tipificadas tradicionalmente como femeninas y los alumnos hacia carreras tipificadas como masculinas. Los estudios masculinos poseen mayor prestigio social que los estudios femeninos. Incluso el hecho de que la enseñanza se haya extendido a una buena parte de la población ha dejado de considerarse prestigioso. Se ha producido una devaluación de los diplomas educativos y por eso las mujeres tienen un mayor acceso a ellos (García de León, 1993: 277).

A la hora de elegir estudios universitarios, las mujeres prefieren titulaciones no excesivamente científicas. Sienten inclinación hacia carreras de grado medio (Diplomaturas) y relacionadas con el cuidado de los demás. Prefieren las carreras de Humanidades, Ciencias Experimentales y Ciencias Sociales y Jurídicas. Se identifican poco con los estudios de Ingeniería y Arquitectura (CIDE/Instituto de la Mujer, 2001: 91).

Nuevamente, estas elecciones van a determinar que posiblemente las mujeres desarrollen su trabajo en la Administración Pública, ya que es en el que tiene mayores posibilidades de éxito profesional. La Administración Pública, formalmente, es una organización social igualitaria, en la que existen pruebas objetivas de ingreso (oposiciones). Dentro de la Administración Pública, la mujer ha sentido una inclinación especial por la enseñanza (una vez más comprobamos cómo se reproducen los roles tradicionales femeninos).

Históricamente, las mujeres siempre han estado presentes en la educación, tanto en la escuela como educando a sus hijos. Cuantitativamente, la 
educación es la institución en la que mayor presencia femenina existe. De ahí que se hable de una feminización de la enseñanza. Según las profesoras García González y Blanco García (1993), la feminización de la enseñanza se ha presentado como un "problema", aunque no están claras las razones por las que se ha calificado así. Aquellos que alertan de este peligro consideran que la presencia de mujeres docentes va en detrimento de la calidad de enseñanza y de una buena formación de los alumnos. En esta línea también se argumenta que "las mujeres son más conformistas, más cómodas, tienen menos motivación para el logro, en definitiva porque son el sexo débil y nunca se hacen adultas, responsables, y por ende son poco eficientes en sus trabajos" (García González, Blanco García, 1993:92). Las autoras suponen que esta preocupación va dirigida hacia los alumnos varones. Critican esta postura argumentando que es la propia sociedad la que empuja a las mujeres hacia determinadas profesiones, actividades que, en la mayoría de los casos, son una prolongación de las tareas domésticas y que, en el caso de la enseñanza, es una extensión de la maternidad. Las mujeres componen porcentajes elevadísimos del profesorado de los niveles no universitarios del sistema de enseñanza y descienden notablemente conforme el nivel se eleva. Sin embargo, la participación de las profesoras en los cargos de dirección y gestión de los centros educativos es muy baja.

En los primeros niveles educativos (Educación Infantil y Educación Primaria) su presencia ha sido evidente y significativa. Mientras que en Educación Infantil y en Educación Primaria un 63\% de los docentes son mujeres, en Secundaria suponen poco más del 40\%. A medida que sube el nivel educativo, la presencia de las docentes disminuye; así, en el nivel universitario tres de cada diez docentes son profesoras (CIDE/Instituto de la Mujer, 2001: 111-117)

Observando estos datos, no se puede hablar de forma general de la feminización de la enseñanza en lo que atañe al profesorado, más bien habría 
que hablar de la feminización de las aulas (García de Cortázar; García de León, 1997).

\section{Las mujeres y la Universidad}

Es a finales de la década de los setenta y principios de los ochenta cuando comienzan a trabajar de forma remunerada las primeras mujeres que han estudiado en la Universidad. A partir de este momento se produce una feminización de esta organización social, aunque esta feminización se produce más dentro de las aulas que entre el profesorado, sobre todo determinados escalafones del profesorado.

En los últimos años, el personal docente universitario ha crecido más que el alumnado, pero aún así algo más del 30\% de los docentes son mujeres (García de Cortázar, García de León, 1997: 21)

El número de profesoras universitarias se incrementa a medida que la categoría profesional disminuye: a mayor categoría menos mujeres. Son mujeres jóvenes, lo que quiere decir que han accedido a la carrera universitaria en fechas recientes. Este hecho manifiesta que el poder de decisión en las universidades se concentra en los hombres. Encarna Roca i Tria (1995) en una ponencia titulada "Mujer y poder en la Universidad", presentada en la Universidad de Oviedo, resume la situación de las profesoras universitarias de esta forma. Incluso sugiere la posibilidad de establecer el sistema de cuotas para que las mujeres puedan acceder a los escalafones de poder y prestigio en la Universidad.

El mayor número de profesoras se encuentra en Humanidades y Ciencias Sociales, seguidas de Ciencias de la Salud y Ciencias Experimentales. Sin embargo, la participación femenina en el área de Ingeniería y Tecnología es escasa. 
A pesar de haber más alumnas en las titulaciones tipificadas como femeninas, no significa que haya un porcentaje más alto de profesoras. $\mathrm{Cu}$ riosamente, la discriminación es mayor en las carreras llamadas de letras, las cuales se han nutrido mayoritariamente de mujeres. Para explicar, en buena parte, esta desigualdad, plantemos la hipótesis de una posible influencia de la Iglesia católica sobre los varones. Es decir, en épocas anteriores, durante los años sesenta y setenta, las familias de clase media o alta procuraban enviar a sus hijos e hijas a colegios con cierto prestigio. Estos colegios eran religiosos. Por un lado, las mujeres que estudian en estos colegios pasan, en mayor proporción que las que estudian en los institutos, a la Universidad. Pero llegan a la Universidad con una discriminación "interiorizada". No todas las universitarias pretenden ejercer su carrera. Su futuro y sus perspectivas vitales están en torno al hogar y al matrimonio. Estos estudios superiores que realizan mayoritariamente las mujeres proporcionan una formación cultural más que una formación estrictamente profesional (Carbajo Vázquez, 2003: 235-240). Por otro lado, las familias de clase baja tenían pocas posibilidades de invertir en estudios para sus hijos/as. Generalmente tenían más acceso los chicos que las chicas. En ocasiones, se recurría a enviar a los hijos a los Seminarios. De esta forma éstos podían conseguir una formación que les sirviera para su futuro. Sabida es la preferencia que ha tenido la Iglesia católica española por las disciplinas de Humanidades y Ciencias Sociales. En principio, se preparaba a estos chicos para el sacerdocio, aunque muchos de ellos, con el tiempo, se separaban de él.

Sin embargo, las autoras de Universidades y universitarios (19701990) creen que la Universidad española, tradicionalmente, ha tenido preferencia por los estudios humanísticos porque éste es un país que, en épocas pasadas, estaba escasamente industrializado e incluso no se apreciaban los conocimientos técnicos. Además, señalan, la creación de estudios de Humanidades y Ciencias Sociales ha requerido siempre menos inversión que los de 
carácter técnico (1992: 94-95).

Sin embargo, en el área de Ingeniería y Tecnología se ha producido un mayor dinamismo. Si relacionamos el número de alumnas con el número de profesoras, comprobamos que existe mayor equiparación. Este hecho se debe fundamentalmente a que las arquitectas y las ingenieras tienen más dificultades que sus colegas varones para ejercer la profesión y optan por la docencia ya que encuentran menos trabas (García de Cortázar, García de León, 1997: 21-22).

Si la presencia de la mujer es escasa en el nivel universitario, ésta se encuentra subrepresentada en los escalafones superiores de la pirámide. Las estadísticas de la Universidad española demuestran que alcanzar determinadas posiciones en esta organización social sigue resultando más difícil a las mujeres que a los varones.

Las catedráticas de universidad representan en torno al 10\% del total del profesorado. Este acceso a la cátedra se ha producido fundamentalmente en los últimos años. Los varones cuentan con más experiencia en estos puestos. Quizás ésta es la razón por la que las mujeres han tenido y tienen escasa influencia en las áreas dominadas principalmente por los hombres. Son las áreas de Humanidades y Ciencias Sociales en las que mayor número de catedráticas hay. Pero aún en este caso, las mujeres representan el 16\% y el 11\% del total de catedráticos, respectivamente (García de Cortázar, García de León, 1997: 24-28). Este dato llama más la atención si tenemos en cuenta que para el curso 2000-2001, más del 66\% de los alumnos que finalizaron estudios en el área de Humanidades eran mujeres y más del $65 \%$ en el área de Ciencias Sociales (CES, 3/2003: 64).

Hay que destacar que son las universidades más jóvenes (creadas en los últimos años) y las de mayor tamaño (Madrid, Barcelona) las que cuentan con mayor número de catedráticas que la media nacional. 
Esta escasa presencia de mujeres en los escalafones superiores implica, a su vez, que estén excluidas de las decisiones en la Universidad. Para ser elegido para determinados cargos, por ejemplo el de rector, existe la obligatoriedad de ser catedrático. Puesto que el porcentaje de mujeres que ocupan este estatus es simbólico, la probabilidad de que una de ellas ocupe un cargo de este tipo es mínima (García de León, 1993: 297-298).

Éste es el retrato de la mujer docente universitaria en España, pero no es mucho peor que el que pueda presentar cualquier mujer con las mismas características de cualquier país de la Unión Europea. Para todas las categorías docentes la media de la presencia femenina está en el $26 \%$, mientras que en España es algo mayor (poco más del 30\%). En el conjunto de la investigación, los varones son más de las dos terceras partes del total de investigadores, según datos referidos al año 1999 (CES, 3/2003: 233-236).

Ya en 1988 la Comisión Europea encargada de fomentar la igualdad entre géneros expresó su preocupación por la escasa representación de la mujer en la investigación científica y tecnológica en general, y en la toma de decisiones en particular. El Parlamento Europeo instó a los Estados miembros a que "promuevan acciones positivas para favorecer la presencia de la mujer también en los niveles más altos de las universidades y centros de investigación". En un Seminario Internacional (Women in science: International workshop) que se celebró en Bruselas en 1993, bajo el Proyecto Mujer y Ciencia, se expresó la inquietud debido a la escasez de mujeres en el ámbito científico.

Estas preocupaciones se siguieron debatiendo en la Conferencia Mujeres y Ciencia, organizada por la Comisión Europea, que se celebró en 1998 en Luxemburgo. La Comisión Europea propuso una estrategia coherente dentro del Quinto Programa Marco para promover la investigación por, para y sobre las mujeres. 
Estas recomendaciones están avaladas por estudios realizados por dicha Comisión en los que queda de manifiesto la desigualdad entre géneros. La Comisión denuncia:

1) Son pocas las mujeres que participan en comités científicos importantes y en la toma de decisiones políticas clave.

2) Las mujeres suelen abandonar la vida académica antes de conseguir un cargo.

3) Cuento más alto es un puesto en la jerarquía, menor es el porcentaje de mujeres que lo ocupan.

4) El porcentaje de catedráticas es muy bajo y oscila entre un 5\% de los Países Bajos y el 18\% de Finlandia.

5) No hay variaciones considerables en la proporción de mujeres en las distintas disciplinas.

6) Las disciplinas con menos mujeres en la mayoría de los países suelen ser las más valoradas.

La escasez de mujeres en puestos científicos superiores afecta a las perspectivas de las científicas de alcanzar tales cargos. Cifras recientes muestran un reducido número de mujeres en los puestos directivos de las universidades europeas. En España, en 1999, el 1.6\% de los rectores y el 9.8\% de los vicerrectores eran mujeres. Que las mujeres no ocupen puestos superiores de gestión en las universidades tiene algunas consecuencias: por un lado, las mujeres no asisten a los debates donde se toman las decisiones políticas, por lo que no pueden plantear alternativas a la situación y a los procedimientos actuales; por otro, debido a su escasez, las que entran en el sistema carecen de modelos de referencia (Comisión Europea, 2001: 2-15). 
¿Cuáles son las razones por las que tan sólo una élite de mujeres llega a la cima de la Universidad? Si bien la docencia se ha considerado como "una cuestión de mujeres", sin embargo la Universidad ha sido un espacio ocupado tradicionalmente por varones. Ma Antonia García de León (1993: 297) apunta distintas razones por las que los varones se sienten atraídos por la universidad:

a) Tradicionalmente, el trabajo en la Universidad se ha considerado como ligero y compatible con otro tipo de ocupación. La Universidad ofrece prestigio al profesional. Este es el caso de algunos abogados y médicos.

b) La Universidad se ha prestado a ser un ámbito en el que es fácil el poder académico. Se ha utilizado como un trampolín para alcanzar otras esferas de poder, sobre todo, el espacio político.

c) Aquellos ámbitos en los que hay posibilidades de ejercer el poder o de intercambiar influencias son preferidas por los varones. Y, por el contrario, de estos espacios son excluidas las mujeres o, lo que es más frecuente, ellas mismas se excluyen de un juego de poder que les desfavorece (García de León, 1993).

Concepción Fernández Villanueva (1989: 161) también señala la Universidad como una organización social de gran influencia: tiene el poder de la transmisión del conocimiento, es un ámbito privilegiado de investigación y está muy próxima a la cúpula del poder político. Estas características la hacen muy apetecible a los varones, educados en el objetivo del éxito profesional.

En este mismo sentido también se expresan los autores del artículo "Cambio y desigualdad en el profesorado universitario". Se preguntan por qué tradicionalmente la Universidad ha atraído a los varones cuando, por el contrario, no han aceptado trabajar en los niveles inferiores de la educación. Consideran que el trabajo en la Universidad, por un lado, ha sido compatible con otra actividad y además confería prestigio. Por otro lado, la Universidad 
se ha prestado a ser utilizada como ejercicio del poder académico o como un trampolín para saltar a otros ámbitos de poder, especialmente el poder político. Estos autores hablan de la Universidad como un "universo masculinizado" (1994: 136).

En una investigación realizada en la provincia de León, Las mujeres y el poder en las organizaciones educativas" (Díez Gutiérrez, Terrón Bañuelos, Valle Flórez, Centeno Juárez, 2003) se pone de relieve que, incluso, en la educación primaria y secundaria, cuanto más grande es el centro y con mayor número de alumnos y profesores, mayor es la frecuencia de que en las tareas de dirección se encuentren un hombre. También se señala que los varones tienen preferencia por dirigir centros de educación secundaria frente a los de educación primaria. Los autores de esta investigación creen que hay relación entre el tamaño del centro de enseñanza y la imagen social de poder que se tiene de ellos. Es decir, "los hombres tienden a competir por las profesiones más prestigiosas y lucrativas” (Díez Gutiérrez, Terrón Bañuelos, Valle Flórez, Centeno Juárez, 2003: 45).

Algunas profesoras dicen que cuando el puesto de trabajo está poco remunerado o bien ha perdido prestigio social, deja de ser apetecido por el hombre y su hueco es ocupado automáticamente por una mujer. Es lo que se ha dado en llamar "política de tierra quemada". Y por el contrario, cuando el puesto está ganando prestigio, menos mujeres consiguen avanzar en él (Roca i Tria, 1995).

Otra de las razones que se apuntan para explicar que la mujer se limite a ocupar los puestos docentes de nivel inferior y no acceder a puestos superiores es que éste es su segundo trabajo. Ellas deben combinar y hacer compatibles diferentes itinerarios vitales y la carrera universitaria está pensada para los varones. Es difícil para una mujer ajustar su vida familiar, su maternidad, a su carrera profesional. Los requisitos para la obtención de una cátedra de universidad están diseñados por y para varones y las mujeres deben realizar 
un mayor esfuerzo y renunciar, en algunos casos, a otras facetas de sus vidas (Mari-Klose, Nos Colom, 1999: 11).

Aunque, aparentemente, la sociedad actual acepta la incorporación laboral de la mujer, se siguen transmitiendo una serie de estereotipos que culpabilizan a las mujeres que desarrollan una actividad en el ámbito público. Este trabajo se califica de "abandono familiar". (Alberdi, 1999: 234). La dedicación a la familia no debe quedar condicionada por los intereses laborales de la mujer. Sus oportunidades de promoción laboral, que en muchos casos supone una cierta movilidad geográfica, están supeditadas a su función "principal": ser madre y esposa (CECS, 1999: 171). Las tareas extradomésticas son voluntarias para las mujeres. De esta forma encontramos connotaciones sociales distintas cuando valoramos el trabajo masculino (entrega y sacrificio para mantener a la familia) y el trabajo femenino ("las mujeres trabajan para ellas y los hombres trabajan para su familia”, Alberdi, 1999: 234).

Es interesante el análisis que hace Lucila Finkel (1999) sobre "profesión y género". Considera que las barreras legales que impiden a las mujeres desempeñar una profesión han desaparecido, pero que existen formas muy sutiles de discriminación que impiden su desarrollo profesional. Propone tres tipos de explicaciones por las que se puede entender por qué las mujeres no pueden romper el "techo de cristal". En primer lugar, se trata de las diferentes formas de socialización de niños y niñas. Esta diferente forma de socializar influye, por un lado, en la orientación de estudios y en las expectativas profesionales. Por otro en la desigual distribución del trabajo doméstico que hace que las mujeres sigan siendo mayoritariamente responsables de la familia y combinan el trabajo remunerado con las tareas domésticas. Esto supone que las mujeres están en desventaja con respecto a sus colegas, ya que carecen de la ayuda "de detrás del telón" y no disfrutan de los beneficios de la carrera "de dos personas" en la que está implicada la pareja. 
En segundo lugar, está la discriminación institucional hacia las mujeres. A medida que se asciende en la escala jerárquica de las organizaciones, se debe dedicar más tiempo y esfuerzo por parte del trabajador. Esto supone llevar trabajo a casa, trabajar más horas, viajar, etc. Las mujeres trabajadoras deben hacer frente a estos requerimientos por parte de la empresa y a las necesidades de sus familias. Además, la mayor dedicación al trabajo se realiza entre los 25 y 35 años, período que suele coincidir con los años de crianza de los hijos.

Por último, desde una perspectiva marxista-feminista, el origen de la discriminación está en un sistema de tipo patriarcal, por el cual los hombres asignan a las mujeres ocupaciones o profesiones muy específicas. Para ello se pueden utilizar cuatro posibles estrategias de cierre social: estrategias de exclusión y demarcación, mediante las cuales los grupos masculinos dominantes confinan a las mujeres en actividades muy concretas y con menos reconocimiento social y permanecen en ellas bajo el control masculino. Estrategia de inclusión, utilizada por las mujeres para contrarrestar las dos anteriores y que fuerza la entrada de las mujeres en una profesión. Una forma de acceder es mediante las credenciales universitarias. Y, por último, el doble cierre, mediante el cual se protegen todas las actividades de una profesión del intrusismo exterior (1999: 209-211).

Si todavía existe resistencia al trabajo femenino fuera del hogar, mayor es la resistencia en lo que respecta al reparto de tareas dentro de la familia y más concretamente en lo que tiene que ver con el cuidado y educación de los hijos. Durante el año 2001, las mujeres dedicaban mucho más tiempo que los hombres al trabajo doméstico y menos al trabajo remunerado, al ocio y al estudio. El tiempo dedicado, tanto por hombres como por mujeres, a las "necesidades personales" es exactamente el mismo, 10 horas 34 minutos, respectivamente (Instituto de la Mujer, 2001: 13). 
Las grandes diferencias entre mujeres y hombres, respecto al tiempo dedicado al trabajo doméstico, se deben, fundamentalmente, al tiempo empleado en el trabajo de la casa propiamente dicho (cocinar, fregar, barrer, quitar el polvo, lavar la ropa, planchar, tender, coser...). Las mujeres dedican a estas tareas cinco veces más tiempo que los hombres.

Las mujeres también dedican más tiempo que los hombres al cuidado de la familia (mientras que ellas usan 1 hora 51 minutos de su tiempo diario, ellos emplean 51 minutos) y a las compras (Instituto de la Mujer, 2001: 1314).

En la mayoría de los casos, la mujer no se siente acompañada por su pareja en su trayectoria profesional. Pocos hombres anteponen el desarrollo profesional de su mujer al suyo propio, mientras la situación contraria es la predominante. Así tenemos que mientras el "proyecto profesional" del hombre acaba siendo un "proyecto familiar", el de la mujer es suyo en exclusiva, aunque encuentre, en algunos casos, comprensión y ayudas puntuales (CECS, 1999: 173).

\section{Las mujeres docentes en la Universidad de León}

En la Universidad de León, durante el curso 2003-2004 se encuentran pocas mujeres como docentes, un $38,84 \%$ del total de docentes. En relación al conjunto de las universidades españolas, un 33\%, podemos decir que la presencia de mujeres en esta universidad es algo mayor (CES 3/2003: 233).

Comprobamos que a mayor nivel educativo, menor número de mujeres en la docencia. Según datos de la Dirección Provincial de Educación de León, para el año 2001 las mujeres suponen más del 70\% de los docentes en las etapas de educación infantil, primaria y educación especial (Díez Gutié- 
rrez, Terrón Bañuelos, Valle Flórez, Centeno Suárez, 2003: 4).

Si analizamos la distribución de profesores y profesoras según la categoría docente que ocupan, nuevamente apreciamos un desigual reparto, sobre todo en los puestos de la cima de la pirámide. Recordemos que estos puestos son de gran importancia en la carrera docente universitaria puesto que son los que permiten acceder a los cargos de gestión, poder y responsabilidad (Rectorado, Decanatos, Direcciones de Departamento).

Tabla 1: No PROFESORES Y PROFESORAS POR CATEGORÍA

\begin{tabular}{|l|r|r|}
\hline CATEGORÍA & H & M \\
\hline Catedráticos de Universidad & 96 & 18 \\
\hline Profesores Titulares de Universidad & 162 & 135 \\
\hline Catedráticos de Escuela Universitaria & 44 & 24 \\
\hline Profesores Titulares de Escuela Universitaria & 71 & 73 \\
\hline Ayudantes de Universidad (LRU) & 2 & 4 \\
\hline Ayudantes de Escuela Universitaria (LRU) & 10 & 14 \\
\hline Ayudantes & 17 & 16 \\
\hline Profesores Asociados a Tiempo Completo & 12 & 18 \\
\hline Profesores Asociados a Tiempo Parcial & 157 & 64 \\
\hline Profesores Eméritos & 5 & \\
\hline Profesores Visitantes & 2 & 1 \\
\hline
\end{tabular}

Fuente: Universidad de León. Marzo, 2004.

De las 114 cátedras ocupadas en esta Universidad tan sólo 18 son poseídas por mujeres $(15,79 \%)$. Esta cifra es tres puntos superior a la media nacional, lo cual no indica que la situación en esta Universidad sea mejor. Las profesoras titulares de universidad representan un $45,44 \%$ de esta cate- 
goría, lo que supone una participación femenina paritaria. Este dato es muy significativo si tenemos en cuenta que esta participación es prácticamente diez puntos superior a la media nacional (CES 3/2003: 232). Otras categorías con representación femenina paritaria son Titular de Escuela Universitaria (50,68\%) y Ayudantes (53,97\%). Dentro de la categoría de Ayudantes hay que destacar la paridad entre los Ayudantes de la Ley de Ordenación Universitaria (LOU) que entró en vigor en el año 2001.

Este es un curso académico en el que todavía conviven figuras docentes de la Ley de Reforma Universitaria (LRU), aprobada en 1983, con las actuales. Incluso, a comienzos de este año, hemos asistido al concurso de algunas plazas que se han cubierto bajo el ámbito de la vieja ley. En esta Universidad, excepto la categoría de Ayudante, no encontramos otras categorías reconocidas y evaluadas bajo los criterios de la LOU.

Los escalafones docentes feminizados son: Ayudantes de Universidad (LRU), Ayudantes de Escuela Universitaria (LRU) y Profesores Asociados a Tiempo Completo $(66,67 \%, 58,32 \%$ y $60 \%$, respectivamente).

Las profesoras están escasamente representadas en los estratos de Catedráticos de Universidad, Catedráticos de Escuela Universitaria, Profesores Asociados a Tiempo Parcial, Profesores Eméritos y Profesores Visitantes $(15,79 \%, 35,78 \%, 28,96 \%, 0 \%$ y $33,33 \%$, respectivamente).

De todo el personal docente de la Universidad tan sólo el 26,45\% de los funcionarios son mujeres, mientras que están en esta situación el 39,46\% de los profesores. Estos datos, una vez más, indican la desigualdad existente en una organización social que aparenta la no discriminación por razón de sexo.

Efectivamente, del 34,08\% del personal docente contratado, el 63,65\% son varones. Esto viene explicado por la gran afluencia de Profesores Asociados a Tiempo Parcial. Los Profesores Asociados son contratados tempo- 
ralmente por la Universidad para cubrir las necesidades docentes de cada momento. Tanto la LRU como la LOU reconocen en esta figura a aquellos especialistas de reconocida competencia que desarrollen su actividad profesional fuera de la Universidad. Esto explica el elevado número de los mismos. Por un lado, las mujeres se han incorporado más tarde al mundo laboral, y en un mundo del trabajo predominantemente masculino llegar a ser una especialista reconocida resulta realmente difícil. Por otro, como ya hemos visto, la "doble jornada" de la mujer profesional hace casi imposible tener tiempo para otra ocupación.

Esta última razón también corresponde al hecho de que dentro de la categoría de Profesores Eméritos todos sean varones. Los hombres planifican su vida en base a su profesión, por eso se resisten a dejarla mucho más que las mujeres. Éstas han llenado la suya de otras actividades que también les han resultado satisfactorias.

El hecho de que en la Universidad de León haya mayor proporción de mujeres docentes que en la media nacional puede deberse a que esta es una Universidad joven. Durante este año 2004 de celebra su $25^{\circ}$ aniversario.

Si hacemos un análisis de las cátedras de Universidad comprobamos que el reparto entre profesores y profesoras es desigual. No sólo se trata de una discriminación en cuanto al número de mujeres que ocupan este estatus, sino también la tardía incorporación que han hecho en relación a sus compañeros.

La Universidad de León nació por Ley 29/1979. Durante los años 1980, 1981, 1982 y 1983 sólo había profesores catedráticos. La primera cátedra que ocupa una mujer es en el año 1985, siendo pionera en esta universidad. La diferencia es sorprendente, mientras que las cátedras que se inauguran en 1980 las ocupan 35 profesores, en 1985 tan sólo lo hace una mujer. 
Si tenemos en cuenta el número de catedráticos y los años que lleva funcionando la Universidad, comprobamos que cada año una media de 3,84 hombres toman posesión de su cátedra. Sin embargo, si realizamos el mismo cálculo para las mujeres, constatamos que la media es de 0,77. Es decir, ni siquiera una mujer por cada año de existencia de la Universidad ha tomado posesión como catedrática. Las cifras hablan por sí solas.

Tabla 2: Proporción de catedráticos y catedráticas por departamento.

\begin{tabular}{|c|c|c|c|}
\hline DEPARTAMENTO & Catedráticas & Catedráticas & TOTAL \\
\hline $\begin{array}{l}\text { Higiene y tecnología de los } \\
\text { alimentos }\end{array}$ & $60 \%$ & $40 \%$ & 100 \\
\hline $\begin{array}{l}\text { Patrimonio histórico artístico y } \\
\text { cultura escrita }\end{array}$ & $33,33 \%$ & $66,67 \%$ & 100 \\
\hline Ciencias Jurídicas & $25 \%$ & $75 \%$ & 100 \\
\hline $\begin{array}{l}\text { Filosofía y Ciencias de la } \\
\text { Educación }\end{array}$ & $33,33 \%$ & $66,67 \%$ & 100 \\
\hline $\begin{array}{l}\text { Ecología, genética y } \\
\text { microbiología }\end{array}$ & $20 \%$ & $80 \%$ & 100 \\
\hline Biología animal & $25 \%$ & $75 \%$ & 100 \\
\hline Patología animal & $9,09 \%$ & $90,91 \%$ & 100 \\
\hline $\begin{array}{l}\text { Farmacología, toxicología, } \\
\text { enfermedades y fisioterapia }\end{array}$ & $50 \%$ & $50 \%$ & 100 \\
\hline Derecho Privado & $50 \%$ & $50 \%$ & 100 \\
\hline $\begin{array}{l}\text { Derecho de la Administración y } \\
\text { Relaciones Internacionales }\end{array}$ & $14,28 \%$ & 85,72 & 100 \\
\hline Bioquímica y biología molecular & $20 \%$ & $80 \%$ & 100 \\
\hline Fisiología & $33,33 \%$ & $66,67 \%$ & 100 \\
\hline
\end{tabular}

Fuente: Universidad de León. Marzo, 2004. Elaboración propia. 
Las catedráticas están adscritas a los siguientes departamentos, en orden decreciente de representación: Higiene y Tecnología de los alimentos; Farmacología, toxicología, enfermedades y fisioterapia; Derecho Privado; Patrimonio histórico artístico y cultura escrita; Filosofía y Ciencias de la Educación; Fisiología; Ciencias Jurídicas; Biología animal; Ecología, genética y microbiología; Bioquímica y biología molecular; Derecho de la Administración y Relaciones Internacionales; y, por último, Patología animal.

Sorprende el hecho de que las catedráticas estén adscritas a doce departamentos, cuando la Universidad cuenta con treinta y tres. Es decir, las profesoras catedráticas realizan sus funciones en el 36,36\% de los departamentos.

Los departamentos a los que pertenecen las catedráticas recogen las áreas de conocimiento ligadas a los estudios de Veterinaria, Biología, Derecho y Filosofía y Letras. Estas titulaciones son las de mayor tradición en la Universidad, dado que ya existían en la ciudad de León, aunque dependientes de la Universidad de Oviedo.

Es importante señalar que la Facultad de Veterinaria fue el primer centro de enseñanza superior y data del curso 1943-44, cuando se integra como facultad en la Universidad de Oviedo.

Los estudios de Biología inician su actividad en el curso 1968-69, convirtiéndose en Facultad en 1980-81.

El colegio universitario de Derecho de León se crea en 1972, y comienzan las clases en la Facultad en 1980-81.

En 1972 también inicia su andadura el Colegio Universitario de Filosofía y Letras. Contaba con dos secciones: Geografía e Historia y Filología (Cordero del Campillo, 1990). 
Este escaso número de docentes catedráticas y el hecho de ser recién incorporadas, hace que los órganos de gobierno de la Universidad prescindan de ellas. Como se ha señalado, éste es un inconveniente para las propias mujeres, ya que cuando están ausentes de las estructuras de gestión y poder, las decisiones tomadas no tienen en cuenta la presencia femenina. Un ejemplo de ello puede ser el organigrama de Rectorado. En este momento, el equipo rectoral está en funciones. Recientemente ha habido elecciones y aunque continúa en el cargo de rector el mismo varón, es posible que haya modificaciones en el resto del equipo.

La cima de la pirámide está presidida por un catedrático. La ley determina que sólo los/as catedráticos/as puedan presentarse para este cargo. Existe muy poca probabilidad de que este puesto sea ocupado por una mujer, dado que en esta Universidad la representación femenina en las cátedras no llega al 16\%. Además, los catedráticos, antiguos en el estatus, han establecido amplias redes de contacto. Son muchos y se apoyan mutuamente. El rector cuenta con un equipo encargado de los ocho Vicerrectorados. Tan sólo uno de éstos está encabezado por una mujer, y es el de Estudiantes y Asuntos Sociales. No puede ser casualidad que hayan puesto al frente de este vicerrectorado a una mujer. De alguna forma se cree que las mujeres están más capacitadas para atender, cuidar y velar por los intereses de los más jóvenes.

Cada Vicerrectorado cuenta con una serie de Secretariados, su número varía en función de las necesidades. Podría parecer que aquí abundarían las mujeres. Después de todo son tareas dependientes del Vicerrector. Pero no es así. Si hay un total de 29 Secretariados, tan sólo el 32,14\% son ocupados por mujeres. Nuevamente, el mayor número de Secretariados ocupados por mujeres son los pertenecientes al Vicerrectorado de Estudiantes y Asuntos Sociales.

En cuanto a lo que se refiere a las direcciones de Departamento, observamos que de los 33 departamentos que conforman esta Universidad, tan sólo 
8 están dirigidos por mujeres. Es decir un $24,24 \%$ de los departamentos tiene a una profesora al frente. Debemos recordar que algunas de estas direcciones se han elegido de acuerdo a la LRU (1983) en la cual se determinaba en el artículo octavo del Título Primero que "la dirección de cada Departamento corresponderá a uno de los catedráticos, y, de no haber candidato de esa categoría, a uno de sus profesores titulares". Esto puede explicar la escasa presencia femenina en las direcciones de Departamento, puesto que el número de catedráticas en la Universidad de León es escaso.

Sin embargo, la Ley Orgánica de Universidades establece en el artículo 25 del Título III del capítulo I que "los directores de Departamento serán elegidos entre profesores doctores pertenecientes a los cuerpos docentes universitarios miembros del mismo. En su defecto podrán ser Directores funcionarios de los cuerpos docentes universitarios no doctores o profesores contratados doctores".

No podemos predecir que esta nueva ley beneficie a las docentes, en cuanto a ocupar cargos de gestión académica. Más bien nos inclinamos a pensar que estos puestos son tan apetecidos por los profesores que ya tienen establecidas redes de confianza, de amistad y de complicidad. Ello implica que las mujeres seguirán estando alejadas de las relaciones políticas universitarias.

Si analizamos la participación de las profesoras en las cúpulas de los centros universitarios, comprobamos que la situación es peor, si cabe. La Universidad de León cuenta con 13 centros universitarios: 8 son Facultades y 5 son Escuelas Universitarias. Las Facultades son las siguientes: Veterinaria, Ciencias Biológicas y Ambientales, Derecho, Filosofía y Letras, Ciencias Económicas y Empresariales, Ciencias del Trabajo, Educación y Ciencias de la Actividad Física y del Deporte. Las Escuelas Universitarias son las siguientes: Ingeniería Industrial e Informática, Ingeniería Técnica de Minas, Escuela Superior y Técnica de Ingeniería Agraria, Ciencias de la Salud y Trabajo So- 
cial. Pues bien, tan sólo uno de estos centros está dirigido por una mujer: la Facultad de Ciencias Económicas y Empresariales.

Nos preguntamos qué requisitos deben reunir los candidatos para cubrir los puestos de Decano de Facultad y Director de Escuela. Nuevamente, recurrimos a las dos últimas leyes de regulación universitaria, puesto que en la actualidad aún existen figuras elegidas bajo los criterios de la antigua ley.

El artículo 21 del Título Segundo de la Ley de Reforma Universitaria establece que los Decanos y Directores "serán elegidos entre Catedráticos o Profesores Titulares del Centro respectivo".

Está claro que la LRU dotaba de gran poder a los catedráticos, estatus ocupado por un número limitado de mujeres, lo que repercute en su escasa representación en el ámbito de responsabilidad y decisión.

El artículo 24 del Título III de la LOU establece que

los Decanos de Facultad y Directores de Escuela serán elegidos entre profesores doctores pertenecientes a los cuerpos docentes universitarios adscritos al respectivo centro.

En su defecto, en las Escuelas Universitarias y en las Escuelas Universitarias Politécnicas, el Director será elegido entre funcionarios de cuerpos docentes universitarios no doctores o profesores contratados doctores.

El 50\% de los Vicedecanatos están ocupados por profesoras, mientras que sólo están el 12,5\% de las Secretarías de las Facultades. Me gustaría destacar el caso de la Facultad de Filosofía y Letras, la cual imparte estudios estereotipados como femeninos, que no tiene ninguna docente en su Decanato.

En cuanto a las Escuelas Universitarias, constatamos el mismo panorama. Ninguno de estos centros tiene una mujer como Directora. El 30\% de 
las Subdirecciones son femeninas, mientras que en el puesto de Secretarias sólo hay un $20 \%$.

En este análisis encontramos hechos que sobresalen de los demás. La Escuela Universitaria de Ingeniería Técnica de Minas está dirigida exclusivamente por profesores. Confirma el estereotipo tradicional: estudios masculinos. Sin embargo, sorprende que la Escuela Superior y Técnica de Ingeniería Agraria no cuente con ninguna profesora en su dirección. Dentro de los estudios de Ingeniería, ésta es la que cuenta con más alumnas.

\section{Conclusión}

A pesar de los esfuerzos que hacen las mujeres por tener un hueco en el mundo laboral, todavía siguen sufriendo discriminación. En el caso que nos ocupa, ésta se produce de forma sutil, pero tiene los mismos efectos. Quizás deberían adoptarse iniciativas con el fin de incrementar la presencia de las mujeres docentes e investigadoras en la Universidad, para paliar de alguna forma el desequilibrio existente en el ámbito de la participación política universitaria. Una representación equilibrada de las mujeres supone que haya no menos de $40 \%$ y no más de $60 \%$ en todos los órganos de toma de decisiones.

\section{BIBLIOGRAFÍA}

Alberdi, I. (1999). La nueva familia española. Madrid: Taurus.

Almarcha Barbado, A.; González Rodríguez, B.; González Jorge, C. (1994). Cambio y desigualdad en el profesorado universitario. En Reis, 66.

Ballarín Domingo, P. (2001). La educación de las mujeres en la España contemporánea (siglos XIX - XX). Madrid: Síntesis. 
Bendix, R.; Lipset, G. M. (1980). "La movilidad social en la sociedad industrial”, en Gras, A.: Sociología de la Educación. Madrid: Narcea, S.A. de ediciones.

Boletín Oficial del Estado, n 307. Lunes 24 de diciembre de 2001.

Bown, G.; Brady, C. (1993). Llegando a la cima. Barcelona: Ediciones Ganica.

Carbajo Vázquez, J. (2003). "Mujeres y Educación (1965-1975)” en Cuesta Bustillo, J. (dir.) (2003). Historia de las mujeres en España. Siglo XX. Tomo II, cap. 16. Madrid: Instituto de la Mujer.

Centro de Estudios del Cambio Social (CECS) (1999). Informe España 1998. Una interpretación de la realidad. Madrid: Fundación Encuentro.

CIDE/Instituto de la Mujer (2001). Las mujeres en el sistema educativo. Madrid: Instituto de la Mujer.

Clair, R. (ed.) (1996). La formación científica de las mujeres. ¿Por qué hay tan pocas cientificas? Madrid: Los Libros de la Catarata.

Comisión Europea (2001). Política cientifica de la Unión Europea. Promover la excelencia mediante la integración de la igualdad entre géneros. Luxemburgo: Oficina de Publicaciones Oficiales de las Comunidades Europeas.

Consejo Económico y Social (2003). Segundo Informe sobre la situación de las mujeres en la realidad sociolaboral española. Madrid: Colección Informes CES. No 3/2003.

Cordero del Campillo, M. (1990). Universidad de León. El primer decenio 1979-1989. León: Secretariado de Publicaciones.Universidad de León.

Díez Gutiérrez, E. J.; Terrón Bañuelos, E.; Valle Flórez, R.E.; Centeno Juárez, D. (2003). Las mujeres y el poder en las organizaciones educativas. Organización y Gestión Educativa. Mayo-Junio (3). ISSN: 11340312. 
Ealy, D.C. (1998). La creatividad en la mujer. Barcelona: Ediciones B, S.A. EL PAÍS, 5-X-2003.

Falcón, L. (1991). Violencia contra la mujer. Madrid: Vindicación feminista. Fernández Villanueva, C. (1989). "Las mujeres en la Universidad española: docencia, investigación y poder. Datos y aspectos cualitativos". En Revista de Educación, n $^{\circ} 290$.

Fernández Villanueva, C. (1996). “Mujer y Educación”, en Blanco García, A. I. (comp.). Mujer, violencia y medios de comunicación. León: Secretariado de Publicaciones, Universidad de León.

Fernández Villanueva, C.; Domínguez Bilbao, R.; Revilla Castro, J.C.; Anagnostou, A.; Sancho Hernández, M. (2003). La igualdad de oportunidades. Los discursos de las mujeres sobre avances, obstáculos y resistencias. Barcelona: Icaria.

Finkel, L. (1999). “¿Qué es un profesional? Las principales conceptualizaciones de la Sociología de las profesiones". En Castillo Mendoza, C.A. Economía, organización y trabajo. Un enfoque sociológico. Madrid: Ed. Pirámide.

Frutos Balibrea, M.D. (1993). "Variables claves para la reflexión en sociología del género: la educación y el trabajo femenino. Su inclusión en los currículos de sociología de la educación”. En Fernández Palomares, F.; Granados Martínez, A. (coords). Sociología de la Educación. Viejas y nuevas cuestiones. Actas de la III Conferencia estatal de Sociología de la Educación. Málaga: Editorial Clave.

García de Cortázar, M.L.; García de León, M.A. (1997). “Mujeres en minoría. Una investigación sociológica sobre las catedráticas de universidad en España”. En Opiniones y Actitudes, n 16. Madrid: CIS.

García de Enterría, E.; Escalante. J.A. (1987). Legislación Administrativa Básica. Madrid: Editorial Civitas. 
García González, M. J.; Blanco García, A.I. (1993). "El problema de la feminización de la enseñanza”. En Juidías Barroso, J.; Loscertales Abril, F. El rol docente. Un enfoque psicosocial. Sevilla: Muñoz Moya y Montraveta Editores.

García de León, M. A. (1990). "Las profesoras universitarias: el caso de una élite discriminada”. En Revista Complutense de Educación. Vol. 1 (3). Madrid.

García de León, M.A.; García de Cortázar, M. L. (1992). Universidades y universitarios (1970-1990). En Revista de Educación. Número extraordinario.

García de León, M. A.; De la Fuente, G.; Ortega, F. (eds.) (1993). Sociología de la Educación. Barcelona: Barcanova. Temas Universitarios.

García de León. M. A.; García de Cortázar, M. L.; Ortega, F. (coord.) (1996). Sociología de las mujeres españolas. Madrid: Editorial Complutense.

García de León, M. A. (2002). Herederas y heridas. Sobre las élites profesionales femeninas. Madrid: Ediciones Cátedra, Colección Feminismos.

Giddens, A. (1998). Sociología. Madrid: Alianza Editorial, Ciencias Sociales.

González Blasco, P.; González-Anleo, J. (1993). El profesorado en la España actual. Informe sociológico sobre el profesorado no universitario. Madrid: Fundación Santa María. S.M.

Instituto de la Mujer (2001). Las mujeres en cifras. 1996-2000. Madrid: Ministerio de Trabajo y Asuntos Sociales.

(2001b). Las académicas. (Profesorado universitario y género). Madrid: Ministerio de Trabajo y Asuntos Sociales.

Kohn, M. L. (1971). "Relaciones paterno-filiales y clase social”. En Fernández Enguita, M. (ed.) (1999). Sociología de la Educación. Barcelona: Ariel Referencia S.A. 
Lawton, D. (1980). "Clase social, lenguaje y educación: revisión crítica de las tesis de Brasil Bernstein”. En Gras, A.: Sociología de la Educación. Madrid: Narcea S.A. de ediciones.

Macionis, J. J.; Plumer, K. (1999). Sociología. Madrid: Prentice Hall.

Mari-Klose, M.; Nos Colom, A. (1999). "Itinerarios vitales: educación, trabajo y fecundidad de las mujeres". En Opiniones y Actitudes, $\mathrm{n}^{\circ} 27$. Madrid: CIS.

Marqués, J.V.; Osborne, R. (1991). Sexualidad y sexismo. Madrid: Fundación Universidad-Empresa. UNED.

Ministerio de Cultura/Instituto de la Mujer (1985). Mujer y Educación. Primeras Jornadas. Serie Documentos 3. Madrid.

Ortiz Gómez, T.; Becerra Conde, G. (eds.) (1996). Mujeres de ciencias. Mujer, feminismo y ciencias naturales, experimentales y tecnológicas. Granada: Feminae.

Pérez-Díaz, V.; Rodríguez, J.C. (2001). Educación Superior y futuro de España. Madrid: Fundación Santillana.

Quintana Cabanas, J. M. (1989). Sociología de la Educación. Madrid: Dykinson.

Roca i Tria, E. (1995). “Mujer y poder en la Universidad”. Ponencia. Seminario de Estudios de la Mujer. Vicerrectorado de Investigación. Universidad de Oviedo. Oviedo.

Sarasúa, P. (1995). Trabaja, mujer, trabaja. Madrid: Acento Editorial.

Sarries Sanz, L. (1993). Sociología de las Relaciones Industriales en la Sociedad Postmoderna. Zaragoza: Mira Editores.

Tobío Soler, C. (2002). "Políticas públicas y estrategias privadas: la conciliación familia-empleo en España". En El Campo. De las Ciencias y las Artes, $\mathrm{n}^{\circ}$ 139. Servicio de Estudios BBVA. (Encuesta de Compatibilización Familia-empleo).

(2002b). “Conciliación o contradicción: cómo hacen las madres trabajadoras". En Reis 97/02, pp. 155-186. 